\title{
Cell Biology During Infection of Plant Viruses in Insect Vectors and Plant Hosts
}

\author{
Qian Chen and Taiyun $\mathrm{Wei}^{\dagger}$ \\ Fujian Province Key Laboratory of Plant Virology, Institute of Plant Virology, Fujian Agriculture and Forestry University, Fuzhou, \\ Fujian 350002, PR China
}

Accepted 11 September 2019.

\begin{abstract}
Plant viruses typically cause severe pathogenicity in plants, even resulting in the death of plants. Many pathogenic plant viruses are transmitted in a persistent manner via insect vectors. Interestingly, unlike in the plant hosts, persistent viruses are either nonpathogenic or show limited pathogenicity in their insect vectors, while taking advantage of the cellular machinery of insect vectors for completing their life cycles. This review discusses why persistent plant viruses are nonpathogenic or have limited pathogenicity to their insect vectors while being pathogenic to plants hosts. Current advances in cell biology of virus-insect vector interactions are summarized, including virus-induced inclusion bodies, changes of insect cellular ultrastructure, and immune response of insects to the viruses, especially autophagy and apoptosis. The corresponding findings of virus-plant interactions are compared. An integrated view of the balance strategy achieved by the interaction between viral attack and the immune response of insect is presented. Finally, we outline progress gaps between virus-insect and virus-plant interactions, thus highlighting the contributions of cultured cells to the cell biology of virus-insect interactions. Furthermore, future prospects of studying the cell biology of virus-vector interactions are presented.
\end{abstract}

Keywords: autophagy, apoptosis, insect cellular ultrastructure, insect immune response, virus-induced inclusion bodies, virus-insect interactions, virus-plant interactions

Plant viruses cause yield loss of crops across the world due to the untreatable damages caused by the virus in plants and the difficulty of preventing viral infection. Such viral infection suppresses plant growth and development, which leads to various symptoms including color change, malformation, local necrosis, and even systematic death. Many pathogenic plant viruses are persistently transmitted via insect vectors, such as whiteflies, leafhoppers, planthoppers, and thrips (Jia et al. 2018). These persistently transmitted viruses are classified into

${ }^{\dagger}$ Corresponding author: T. Wei; weitaiyun@fafu.edu.cn

Funding: This work was supported by the National Key R\&D Program of China (2017YFD0200900), the National Natural Science Foundation of China (31772124), the Natural Science Foundation of Fujian Province (2017J06011), and the Program for New Century Excellent Talents of Fujian Province University (Kla18057A).

The author(s) declare no conflict of interest.

() 2020 The American Phytopathological Society nonpropagative and propagative groups, based on the viral replication or not within the insect vector (Jia et al. 2018). In insects, ingested persistent viruses sequentially access the stylet and esophagus and, then, enter the epithelia cells of the alimentary canal. Propagative viruses replicate and produce progeny viruses in insect bodies. To complete the circulation within insect vectors, the progeny of propagative viruses or nonpropagative viruses spread through insect bodies from the gut, via the hemolymph, to other organs or salivary glands. Finally, virions are ejected with saliva into the plant host during insect feeding (Hogenhout et al. 2008; Wei and Li 2016).

Due to the limited number of proteins encoded by the viral genome, viruses depend on the cellular machinery of their vectors to propagate (Feng et al. 2016; Liu et al. 2015). However, unlike in the plant hosts, such viral propagation leads to nonpathogenic or limited pathogenic responses in the insect vectors. Recent investigations have demonstrated that, once insect vectors perceive invasion of a virus, the defense system restricts viral pathogenicity, either by counterattacking or by compromising the virus (Chen et al. 2017b, 2019b; Huang et al. 2015; Wang et al. 2016).

This review summarizes the mechanisms behind the nonpathogenicity or limited pathogenicity of plant viruses in their insect vectors but showing pathogenicity in the plant hosts. Advances of the interaction between virus and the immune system of insect vectors are summarized, including viral attack causing the formation of inclusion bodies and ultrastructural changes in insects as well as virus-triggered immune responses of the insect vectors, especially autophagy and apoptosis. The corresponding findings of virus-plant interactions were compared. Furthermore, this review presents an integrated view of the balance strategy achieved by the interaction between the persistent plant viruses and insect vectors.

\section{INCLUSION BODIES-THE WEAPON OF VIRAL ATTACK}

Inclusion bodies are key characteristics of virus-induced cytopathology in both insect vectors and plant hosts. The morphologies of these inclusion bodies can be used to determine the taxonomy of viruses. The structures of inclusion bodies and the association of inclusion bodies with viral pathogenicity depend on viral species. Most inclusion bodies are important for the virus to infect its insect vector or plant host and can even act as weapons during viral attack.

Inclusion bodies in the insect vector.

Inclusion bodies induced by persistently transmitted plant viruses are formed in the cytoplasm, nucleus, or cellular junction 
between cells within the insect vectors. The following describes the different morphologies and functions of viral inclusion bodies in insect vectors.

Crystalline arrays of virions. Inclusion bodies in crystalline arrays are a common structure in virus-infected insects and plants. They are formed by aggregating virions and have various sizes and shapes (Jia et al. 2012; Zhao et al. 2019). It is believed that these pools of virions serve as a source for viral infection and guarantee viral titers for the maintenance of persistent infection in insect vectors.

Electron-dense or filamentous inclusion bodies, the sites for viral replication and assembly of progeny virions, namely, viroplasm. In insect vectors, persistent-propagative viruses encode viral proteins to form a specific electron-dense or filamentous viroplasm matrix for efficient viral propagation (Chen et al. 2014; Zheng et al. 2015). Electron microscopy of planthoppers infected with the plant reovirus southern rice black streaked dwarf virus (SRBSDV) (Table 1) shows that the viroplasm matrix contains a granular, electron-dense region and a filamentous region (Mao et al. 2013). The granular viroplasm matrix is the site for the accumulation of viral RNAs and nonstructural proteins P6 and P9-1, encoded by segment 6 and 9 (S6 and S9) of the viral genome, respectively (Mao et al. 2013). The filamentous viroplasm matrix, which accumulates viral RNAs, progeny cores, viral particles, and viral nonstructural proteins P6 and P5 encoded by $\mathrm{S} 5$, is the site where progeny virions assemble (Mao et al. 2013). In contrast, the matrix of viroplasm induced by rice dwarf virus (RDV) (Table 1), which is also a plant reovirus, contains a single granular region that accumulates viral nonstructural proteins Pns6, Pns11, and Pns12 encoded by S6, S11, and S12 of viral genome, respectively (Wei et al. 2006). Pns 12 serves as a scaffold of the viroplasm matrix and orchestrates viroplasm formation via complex networks of interactions among Pns6, Pns11, and core capsid protein P3 of RDV (Chen et al. 2015b). Nonstructural protein NS3 of tenuivirus rice stripe virus (RSV) (Table 1), encoded by the virus-sense open reading frame (ORF) of RNA3, forms the viroplasm matrix to regulate viral replication or assembly by interacting with the filamentous viral ribonucleoprotein in small brown planthopper (SBPH) cells (Ma et al. 2013). Furthermore, rhabdoviruses $\mathrm{N}$ and $\mathrm{P}$ proteins form the viroplasm matrix to support viral replication and the assembly of progeny virions (Gao et al. 2019; Jackson et al. 2018; Yang et al. 2017; Zhao et al. 2019; Zhou et al. 2019). Currently, whether the insect components are involved in the formation of viroplasms induced by plant viruses in insect vectors is unknown.
Fibrillar or tubular inclusions as viral spread vehicles in insect vectors. Current studies suggest that viral nonstructural proteins induced by persistent-propagative viruses could form inclusion bodies with specific fibrillar or tubular structures to serve as the delivery vehicles for viral spread throughout insect vectors. The nonstructural protein NS4 of tenuivirus RSV, encoded by the virus-sense ORF of RNA4, forms cytoplasmic fibrillar inclusions in various tissues of the viruliferous planthopper vector (Wu et al. 2014). These NS4-specific inclusions are closely associated with ribonucleoprotein of RSV via direct interaction between NS4 and the nucleoprotein of RSV for viral spread throughout the bodies of SBPHs (Wu et al. 2014).

Tubule-shaped inclusion bodies in infected cells of insects are a unique feature of most plant reoviruses, e.g., RDV, SRBSDV, and rice gall dwarf virus (RGDV) (Table 1) (Chen et al. 2019a; Wei and Li 2016). These tubular inclusion bodies are assembled by viral nonstructural proteins with selfinteraction and could package virions for cell-to-cell spread within insect vectors. Virion-packaging tubules can pass through multiple tissue and membrane barriers in the insect bodies, including microvilli, intercellular junctional complexes, basal lamina, visceral muscle, the plasmalemma of salivary glands, and even follicular cells of oocytes (Wei and Li 2016). These tubular inclusion bodies protect virions from the immune system of the insect, enabling the virions to spread more effectively, compared with the dissemination of free virions. Actin-associated proteins of the insect vectors can either positively or negatively regulate and facilitate the formation or mobility of tubules in insect vectors (Chen et al. 2015a, 2017a, 2019a; Wei and Li 2016). This particular tubular strategy represents a specific mode of cell-to-cell spread of plant reoviruses within insect vectors.

Fibrillar inclusion targeting and inducing mitochondrial degeneration. Immunoelectron microscopy reveals that the nonstructural protein Pns11 encoded by S11 of RGDV viral genome forms bundles of fibrillar inclusions to target the periphery of degenerated mitochondria in its leafhopper vectors (Chen et al. 2019b). Several RGDV particles are closely associated with free ends of the Pns11 fibrillar structures and can be found along their edges (Chen et al. 2019b; Wei et al. 2011). The single expression of Pns11 in nonhost cells also induces mitochondrial degeneration (Chen et al. 2019b), indicating Pns11 as a pathogenic factor with the inherent ability to target mitochondria.

\section{Inclusion bodies in plant hosts.}

In plant hosts, most inclusion bodies can be found in the epidermal cells and have different morphologies and functions.

Table 1. Viruses addressed in this review

\begin{tabular}{|c|c|c|c|c|}
\hline Virus & Acronyms & Family & Vector & Vector transmission strategy \\
\hline Barley stripe mosaic virus & BSMV & Virgaviridae & - & \\
\hline Cauliflower mosaic virus & $\mathrm{CaMV}$ & Caulimoviridae & Aphid & Nonpersistent \\
\hline Cotton leaf curl Multan virus & CLCuMuV & Geminiviridae & Whitefly & Persistent \\
\hline Cotton leaf curl virus & $\mathrm{CLCuV}$ & Geminiviridae & Whitefly & Persistent \\
\hline Plantago asiatica mosaic virus & PIAMV & Alphaflexiviridae & - & \\
\hline Rice dwarf virus & RDV & Reoviridae & Leafhopper & Persistent-propagative \\
\hline Rice gall dwarf virus & RGDV & Reoviridae & Leafhopper & Persistent-propagative \\
\hline Rice ragged stunt virus & RRSV & Reoviridae & Planthopper & Persistent-propagative \\
\hline Rice stripe virus & RSV & Unassigned & Planthopper & Persistent-propagative \\
\hline Sindbis virus & SINV & Togaviridae & Mosquito & Persistent-propagative \\
\hline Southern rice black streaked dwarf virus & SRBSDV & Reoviridae & Planthopper & Persistent-propagative \\
\hline Tobacco mosaic virus & TMV & Virgaviridae & - & \\
\hline Tomato yellow leaf curl virus & TYLCV & Geminiviridae & Whitefly & Persistent \\
\hline Turnip mosaic virus & TuMV & Potyviridae & Aphid & Nonpersistent \\
\hline Vesicular stomatitis virus & VSV & Rhabdoviridae & - & \\
\hline West Nile virus & WNV & Flaviviridae & Mosquito & Persistent-propagative \\
\hline
\end{tabular}


Similar to insect vectors, plant viruses can induce crystalline arrays of virions or viropalsm in plant hosts. The viroplasms induced by plant reoviruses in support of viral replication and assembly have been well-characterized in plants. The viroplasms induced by the cauliflower mosaic virus (CaMV) (Table 1), a plant pararetrovirus with a DNA genome, appear as analogous electron-dense inclusion bodies and protect viral coat proteins $(\mathrm{CP})$ and virions from the host immunity pathway (Blanc et al. 2014). Furthermore, CaMV also induces the formation of an electron-dense inclusion body that is required for viral transmission by insect vectors, namely, the transmission body (TB) in plant cells (Blanc et al. 2014). Within the TB matrix, viral helper protein $\mathrm{P} 2$ can link viral particles via virion-associated protein P3 (Blanc et al. 2014). During viral transmission by aphid vectors, the aphid stylet penetrates plant cells and, thus, stimulates the disintegration of TBs. This leads to the release of transmissible P2-virus complexes from plant cells into insect stylets (Blanc et al. 2014; Drucker et al. 2002; Martinière et al. 2013). Thus, the viral helper protein recognizes the receptors at the tip of aphid maxillary stylets and causes efficient viral transmission by aphid vectors (Blanc et al. 2014; Drucker et al. 2002; Martinière et al. 2013). Nonstructural proteins of potyviruses can aggregate within infected cells and form cylindrical inclusion bodies, which are considered to be one of the main characteristics of the family Potyviridae (Carrington and Dougherty 1987). The cylindrical inclusion bodies of potyviruses serve as a docking point for viral cell-to-cell movement via plasmodesmata at the cell wall (Deng et al. 2015; Movahed et al. 2017).

\section{ULTRASTRUCTURE CHANGE-THE SKETCH OF A VIRAL ATTACK}

Viral pathogenicity can be diagnosed via changes of cellular ultrastructure, which involves pathological or structural changes of cellular organelles. It is important to clarify the ultrastructural changes caused by plant viruses to better understand viral pathogenesis and the interaction of the virus with its plant hosts. However, studies on virus-induced ultrastructural changes in insect vectors are limited, possibly due to the concept that plant viruses do not negatively impact insect vectors and convergently evolve with them in harmony. This contradicts several insect viruses that normally induce severe cytopathology.

The infection of persistent nonpropagative luteoviruses has been well-characterized to induce the formation of different vesicles in cells of aphid vectors for viral circulation and overcoming barriers in the aphid gut and salivary gland (Gray and Gildow 2003; Gray et al. 2014). In the process of viral entry into gut epithelial cells by endocytosis, luteovirus particles are packaged into coated pits, which bud from the membrane of the apical plasmalemma as virus-containing coated vesicles (Gray and Gildow 2003; Gray et al. 2014). These vesicles transport the viruses to larger endosomes where the luteoviruses are repackaged into tubular vesicles, which then bud off and migrate to the basal plasmalemma via exocytosis (Gray and Gildow 2003; Gray et al. 2014). Similar endocytosis and exocytosis processes occur when luteoviruses infect the accessory salivary glands so that the viruses can be released into the salivary canal (Gray and Gildow 2003; Gray et al. 2014). The tomato yellow leaf curl virus (TYLCV) (Table 1), a geminivirus, has limited pathogenic effects on whitefly vectors. TYLCV infection induces the formation of specific autophagosome with double membranes and autolysosomes with multimembrane structures in the midgut epithelium of whitefly vectors (Wang et al. 2016).
For persistent-propagative viruses, limited pathogenicity has been reported in plant reovirus-infected insect vectors. In cultured cells of leafhoppers, RDV and RGDV can induce viruscontaining autophagosomes with single or double membranes within the cytoplasm, either at the periphery of cell membrane or outside the cells (Chen et al. 2017b). Virus-induced autophagosomes engulf progeny virions in the cytoplasm, but also directly fuse with the plasma membrane to release viral particles (Chen et al. 2017b). Thus, this virus-induced autophagosome appears to be involved in the nonlytic release of viruses from insect cells. In leafhoppers, virus-containing autophagosomes are distributed in the epithelium, microvilli, gut lumen, and visceral muscles (Chen et al. 2017b). Thus, autophagosomes may be a novel machinery to mediate the release of plant reoviruses from the intestinal epithelium of insect vectors.

RGDV infection can also induce ultrastructural changes with the hallmarks of apoptosis in cultured leafhopper cells, such as crescent-shaped nuclei and condensed and marginalized chromatin (Chen et al. 2019b). Apoptotic bodies, which are a typical characteristic of the end-stage of apoptosis, can be found in insect cells and contain virions (Chen et al. 2019b). As mentioned above, RGDV induces the degeneration of mitochondria in cultured leafhopper cells (Chen et al. 2019b; Wei et al. 2011). In the limited area of the intestinal epithelial cells of leafhoppers, similar ultrastructural changes occur, including the reduction and vacuolization of the cytoplasm, the damage to or decrease in number of microvilli, and the appearance of shrunken or crescent-shaped nuclei (Chen et al. 2019b). The mechanisms with which RGDV induces autophagy or apoptosis in leafhopper cells are discussed further below.

The potential pathogenicity of plant viruses becomes severe in small interfering RNA (siRNA) pathway-defective insect vectors. SRBSDV is not pathogenic in its vector, the whitebacked planthopper, nor in a nonvector, the SBPH (Liu et al. 2018). However, knockdown of Dicer-2, a core component of the siRNA pathway, promotes the persistent infection of SRBSDV in both vector and nonvector species and causes severe ultrastructural changes in insect tissues (Lan et al. 2016a; Liu et al. 2018). The observed ultrastructural abnormalities include vacuolization in the cytoplasm, reduction of cytoplasmic density, loss of microvilli brush border integrity, and degeneration of both mitochondria and rough endoplasmic reticulum (ER) (Lan et al. 2016b). If the virus has accumulated beyond the mortality threshold, RGDV infection is lethal (Lan et al. 2016b). Thus, the siRNA pathway in insect vector can modulate persistent infection of plant viruses.

\section{IMMUNE RESPONSE TO VIRUS- COUNTERATTACK OR COMPROMISE}

Plant hosts and insect vectors respond to viruses via immune pathways. The mechanisms for the antiviral immunity of insect vectors involve RNA interference (RNAi), autophagy, and apoptosis. An increasing number of investigations show that autophagy and apoptosis in insect vectors appear to be doubleedged swords in the response to viral infection (Chen et al. 2017b, 2019b; Haxim et al. 2017; Huang et al. 2015; Li et al. 2018; Wang et al. 2016).

\section{Autophagy.}

Autophagy is a cellular process during which cells utilize lysosomes to degrade damaged cellular organelles and macromolecules. This process is regulated by the autophagy-related genes (Atg) to maintain cellular homeostasis. The autophagy machinery also serves as an important innate and adaptive immune response against a variety of viral pathogens (Heaton and Randall 2010). 
The role of autophagy during virus-vector interaction. In insects, an intact autophagic machinery provides an important antiviral cellular response that restricts viral replication via mechanisms such as the selective transferal of viral nucleic acids to endosomal compartments, to stimulate innate immune responses, and the selective degradation of viral proteins (Chiramel et al. 2013; Kudchodkar and Levine 2009). For example, autophagy contributes to the defense against infection by the vesicular stomatitis virus in Drosophila spp. (Table 1) (Nakamoto et al. 2012; Shelly et al. 2009). Recent studies demonstrate the diversity of functions of autophagy during viral infections (Bouley et al. 2014; Carneiro and Travassos 2016; Pei et al. 2014). Autophagy is utilized or modulated by the virus to enhance viral pathogenicity or to regulate innate and adaptive immune responses to viral infection. Autophagy can also promote different stages of viral life cycles, from viral replication to its exit from cells (Carneiro and Travassos 2016; Nakamoto et al. 2012; Pei et al. 2014).

However, the role of autophagy during viral infection is less understood in plant viruses compared with arthropod-borne human viruses. This is partly because established reverse genetic methods or cultured cells are not available for insect vectors. TYLCV infection in whitefly vectors activates the autophagy pathway, which functions as an intrinsic antiviral program and degrades the virus (Wang et al. 2016). TYLCV induces the formation of autophagosomes, which leads to the degradation of the TYLCV CP and its genomic DNA (Wang et al. 2016). The autophagy inhibitors 3-methyladenine or bafilomycin A1, or knockdown of Atg3 or Atg9, increases the viral load and facilitates virus transmission (Wang et al. 2016). The autophagy activator rapamycin notably decreases the amount of viral CP and DNA in the whitefly and inhibits the efficiency of viral transmission (Wang et al. 2016).

In contrast, autophagy in the leafhopper vector compromises the attack of both RGDV and RDV and can even facilitate both viral infection and transmission (Chen et al. 2017b). Insect cells that are infected by these viruses typically form viruscontaining autophagosomes, convert ATG8-I to ATG8-II, and show improved levels of autophagic flux in both the leafhopper intestine and cultured cells (Chen et al. 2017b). Inhibition of autophagy by applying the autophagy inhibitor 3-methyladenine or via knockdown of Atg5 expression significantly blocks viral transmission by insect vectors; however, activation of autophagy via rapamycin or knockdown of Torcl expression increases viral transmission (Chen et al. 2017b). This autophagy-exploiting viral spread may be a general mechanism during the transmission of vector-borne persistent viruses by insect vectors (Fig. 1A).

The role of autophagy in virus-plant interactions. Similar to insect vectors, the role of autophagy in plant hosts also varies by virus and plant species. A key evidence to determine the proviral or antiviral role of autophagy is also the effect of autophagy deficiency on viral infection. Thanks to wellestablished techniques in reverse genetics and gene silencing as well as the acquired understanding of immunity pathways in plants, investigations of virus-induced autophagy in plant hosts are advanced. Most autophagy mechanisms, including the role of autophagy in plants, are understood at the protein level or are based on pathway network analyses. Unlike in insect vectors, the final phenotype and role of autophagy is basically understood.

Targeting viral proteins or components for degradation via the autophagy pathway is a common antiviral mechanism in plants. Li et al. (2018) reported that ATG6 of the plant host restricts the infection of turnip mosaic virus (TuMV) (Table 1), which is a potyvirus, via suppression and, also, likely autophagic degradation of the viral RNA-dependent RNA polymerase (RdRp). The authors further reported that TuMV infection causes ATG6-mediated RdRp degradation (Li et al. 2018). ATG6 targets RdRp of TuMV via direct interaction for autophagic degradation, likely through ATG8, which acts as an adaptor protein. Deficiency of ATG6 or ATG8 enhances RdRp accumulation and promotes viral infection. Overexpression of an ATG6 mutant that binds to RdRp but lacks the ability to mediate RdRp degradation is also reported to inhibit virus replication (Li et al. 2018). This ATG6-RdRp interaction exists
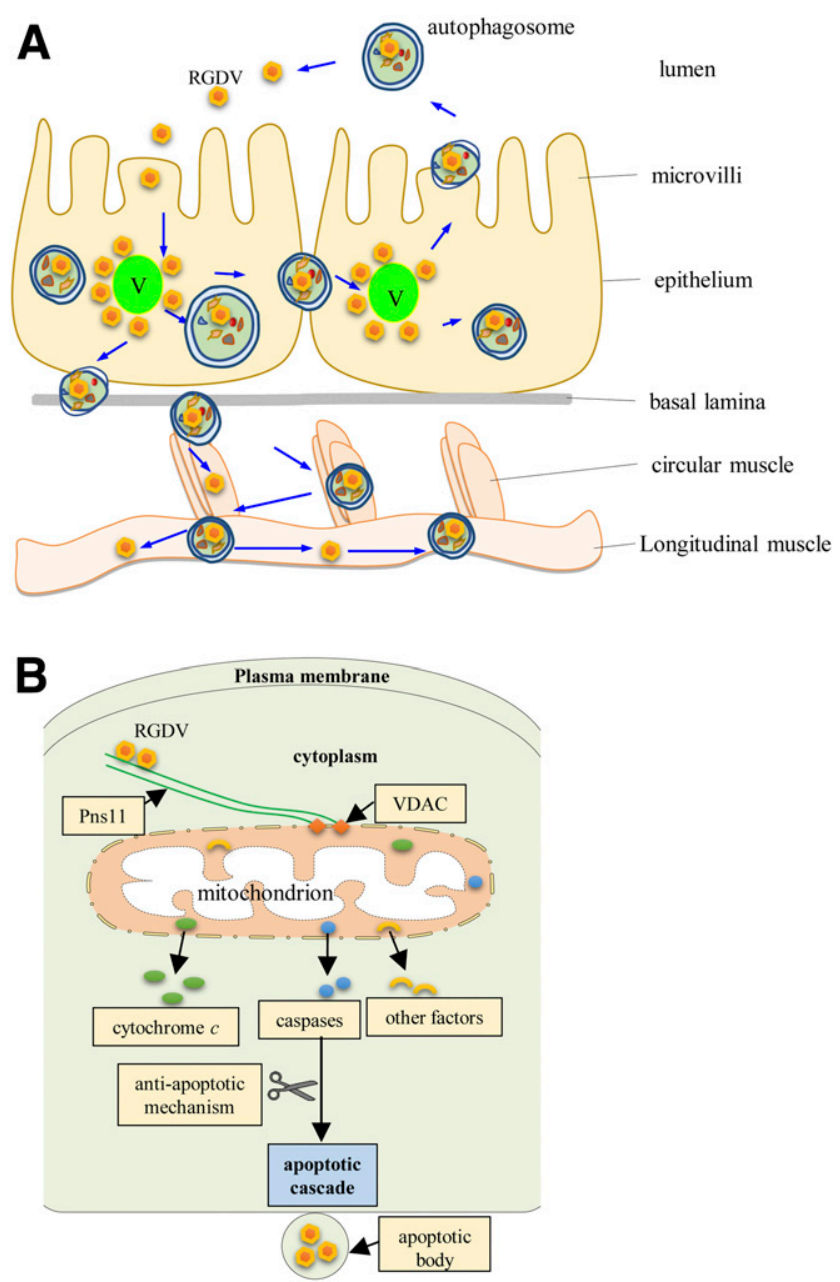

Fig. 1. Proposed model for autophagy and apoptosis triggered by the rice gall dwarf virus (RGDV) in the leafhopper. A, Proviral autophagy triggered by RGDV in leafhopper. RGDV virions enter epithelial cells via receptormediated, clathrin-dependent endocytosis, which is followed by formation of viroplasms for viral replication. Progeny RGDV virions assemble at the periphery of the viroplasms and are engulfed by autophagosomes. These then mediate nonlytic viral release by fusing with the plasma membrane. Such autophagosomes may pass through microvilli, cross to neighbor cells for secondary infection or traverse the basal lamina, and move along visceral muscles that surround the intestinal epithelium. $\mathrm{V}=$ viroplasm. $\mathbf{B}$, Mitochondria-dependent apoptosis triggered by RGDV in leafhopper cells. During early stages after viral replication, the fibrillar structures composed of RGDV Pns11 form. These then target the outer membrane of the mitochondria by directly interacting with voltage-dependent anion channels (VDAC), which leads to the disruption of the outer mitochondrial membrane and the decrease of the mitochondrial membrane potential. The transduction of a series of signals (or potentially other mechanisms) activate capsases, which then trigger the release of cytochrome $c$ and other mitochondrial apoptotic factors from the depolarized mitochondrial membrane into the cytoplasm. Subsequently, downstream events of apoptosis are initiated, including the generation of virion-containing apoptotic bodies. The virus exploits these virus-induced apoptotic events to facilitate infection and transmission by the insect vectors. 
in several RNA viruses ( $\mathrm{Li}$ et al. 2018). The cotton leaf curl Multan virus (Table 1), which is a DNA virus, is targeted by plant autophagy for degradation via interaction of virulence factor $\beta C 1$ and ATG8 (Haxim et al. 2017). The mutant of $\beta C 1$ shows enhanced symptoms and viral DNA accumulation by abolishing its interaction with ATG8. Inhibition of autophagy decreases plant resistance to DNA viruses, while activating autophagy enhances plant resistance against viral infection (Haxim et al. 2017).

In addition to antiviral functions of autophagy described above, the autophagy pathway can be utilized by viruses to promote plant fitness during the viral infection via damaging the immunity of plants, especially the RNAi pathway. The autophagy pathway of the plant host is taken advantage of by the TuMV VPg, which is a potyviral silencing suppressor, to degrade the suppressor of gene silencing 3 for exerting the sabotage of antiviral RNAi of the plant host, thus promoting viral infection (Cheng and Wang 2016). Similarly, the autophagy pathway in plant cells can degrade ARGONAUTE1 (AGO1), which is triggered by viral suppressor of RNA silencing protein P0 from polerovirus (Derrien et al. 2012).

\section{Apoptosis.}

Apoptosis is a normal physiological process of autonomous and programmed cell death (PCD) that is controlled by genes to maintain the stability of the intracellular environment. Apoptosis plays a critical role in embryonic morphogenesis and development, immunity responses against pathogens, cellular damage, and aging (Denton and Kumar 2015; Elmore 2007).

Apoptosis in animals is accompanied by morphological changes, including cellular shrinkage, chromatin condensation, cell fragmentation, generation of apoptotic bodies, and engulfment by phagocytes. In this process, caspases, which form a family of cysteine proteases, are crucial proteases that are responsible for the execution of the apoptotic cascade (Beug et al. 2017).

The role of apoptosis in virus-vector interactions. In general, persistent-propagative viruses induce apoptosis in insect vectors; however, it is typically restricted to a low level to avoid severe damage to insects (Chen et al. 2019b). Within insect vectors, it is pivotal that apoptosis functions as an antiviral mechanism to limit the infection, replication, and dissemination of viruses. For example, apoptosis caused by the West Nile virus (WNV), which is a flavivirus, in mosquitos is proposed to limit the number of WNV-infected cells and inhibits viral infection (Vaidyanathan and Scott 2006). To counter apoptosis, several viruses have evolved specific strategies to inhibit apoptosis or to benefit from apoptosis (Mitchell and Friesen 2012). Insects and cultured insect cells infected by the baculovirus are good models for the study of the underlying mechanisms of antiapoptosis, because viral proteins $\mathrm{p} 35$ and inhibitor of apoptosis (IAP) of the baculovirus have been confirmed to block apoptosis (Clem et al. 1991, 2007). In contrast, the Sindbis virus (SINV) (Table 1), which is an alphavirus, is one of the viruses with the capability to explore apoptosis for replication and dissemination (Griffin and Hardwick 1999; Wang et al. 2012). Although effects of apoptosis induced by viruses in insect vectors have been described and the underlying mechanisms are well-characterized (e.g., the anti-apoptosis mechanism of baculovirus), the general understanding of whether plant viruses can trigger apoptosis in insect vectors as well as the role of apoptosis in viral infection lag behind studies of animal viruses.

The investigations of apoptosis induced by RGDV provide insight into how viruses use apoptosis for both infection and transmission. The nonstructural protein Pns11 is the inducer of an apoptotic response and a pathogenic factor of RGDV. RGDV employs Pns 11 to target the outer membrane of mitochondria, thus causing mitochondrial degeneration and a decrease of membrane potential (Chen et al. 2019b). As a result, the release of apoptosis-related factors triggers an irreversible downstream apoptotic cascade (Fig. 1B). When apoptosis is inhibited via caspase inhibitors or knockdown of caspase gene expression, viral accumulation is blocked (Chen et al. 2019b). In contrast, activation of apoptosis by knockdown of IAP gene expression increases viral accumulation (Chen et al. 2019b). The RGDVinduced apoptotic response benefits viral infection and is restricted to a low level to avoid severe damage to the leafhopper vector to maintain persistent viral infection (Chen et al. 2019b). Another plant virus, the rice ragged stunt virus (Table 1), also induces apoptosis in the salivary glands of the planthopper vector and apoptosis is utilized to release viruses into rice plants (Huang et al. 2015).

The role of $P C D$ in virus-plant interactions. Plants share many hallmarks of apoptosis at the cellular and molecular levels with animals; however, it is controversial whether apoptosis occurs in plant cells. This is because i) the cell walls make the formation of apoptotic bodies impossible, and ii) the absence of phagocytic cells in plants suggests that apoptotic bodies and cell fragments cannot be removed from plants (van Doorn et al. 2011); in addition, iii) plants do not have caspase homologs but, instead, possess an ancestral family of cysteine proteases, the metacaspases, which are essential for PCD (Beug et al. 2017; Sundström et al. 2009; Uren et al. 2000). Thus, most studies still describe this apoptotic-like process as PCD in plants.

PCD maintains homeostasis in organisms and restricts viral infection by sacrificing a small number of cells. Thus, PCD is one of the most effective antiviral responses in plants. Komatsu et al. (2010) reports that the systemic necrosis induced by plantago asiatica mosaic virus infection can be attributed to PCD and the restraint of virus multiplication (Komatsu et al. 2010). Nyalugwe et al. (2016) reports that PCD pathways induced by early plant-TuMV infection can be determined by isolating virulence and stage of infection (Nyalugwe et al. 2016). Despite well-documented PCD studies in plants, the molecular pathways that regulate PCD remain unknown. In fact, the knowledge of PCD in plants is limited due to a lack of homologs of key apoptotic regulators in animals (Kabbage et al. 2017). In contrast, homologs of plant autophagy regulators are relatively well-understood.

PCD is also identified to be associated with hypersensitive response (HR), caused by an invasion of incompatible pathogens (Jan and Khurshid 2013). The transgenic expression of baculovirus p35 protein, which inhibits apoptosis in insects, can suppress the HR triggered by the tobacco mosaic virus (TMV) (Table 1) and causes TMV to spread in a systemic manner in a resistant background (del Pozo and Lam 2003). HR inhibition and TMV systemic spread in p35-expressing plants suggests that animal and plant systems share a common caspase activity against viral infection.

\section{HOW IS PERSISTENT INFECTION OF PLANT VIRUSES ESTABLISHED AND MAINTAINED IN THEIR INSECT VECTORS?}

Except for viruses with limited pathogenic persistence, several persistent plant viruses negatively affect both the behavior and physiology of insect vectors. TYLCV and cotton leaf curl virus (Table 1) reduce the longevity, daily fecundity, and egg hatchability of its insect vectors (Ogada et al. 2012; Rubinstein and Czosnek 1997; Sidhu et al. 2009). RSV and SRBSDV reduce survival of planthopper vectors, longevity, oviposition, 
and egg hatchability (Chen et al. 2016; Lei et al. 2014; Li et al. 2015; Tu et al. 2013; Wan et al. 2015). RDV negatively affects the survival of leafhopper nymphs, intensity of diapauses, fecundity of female adults, and the longevity of adults. Moreover, it causes abnormal cytological structures of a number of internal organs of nymphs and adults (Nakasuji and Kiritani 1970; Nasu 1963). RGDV infection significantly decreases vitellogenin expression in leafhoppers, which adversely affects the development of their oocytes, and, finally, reduces fecundity of leafhopper vectors (Chen et al. 2016). However, adverse effects of these persistent plant viruses are moderate compared with other pathogens that have strong pathogenicity. Far less information is available on the underlying mechanisms of these limited effects.

The remaining question is how persistent viruses with limited pathogenic effects are established and maintained in the insect vector. To discuss possible mechanisms, first, the reasons why these viruses have limited pathogenicity or disadvantage effects are discussed. Using the combination of persistent RGDV and leafhoppers as examples, RGDV causes limited pathogenic and immune response in leafhoppers and these pathogenicity and immune responses are beneficial for viral infection (Chen et al. 2017b, 2019b). In insect vectors, transmission barriers of viruses mainly refer to multiple tissue and membrane barriers at the tissue level (Wei and Li 2016). The limited apoptosis or autophagy in leafhopper vectors may breach tissue and membrane barriers, which is similar to the role of apoptosis in Aedes aegypti infected with SINV (O'Neill et al. 2015). This breach makes it easy for the virus to overcome transmission barriers and infect insect vectors. Regarding the viral life cycle, RGDV modifies the immune response of the insect vector and causes ultrastructural changes for their dissemination and subsequent infection. This is similar to a number of viruses that use apoptosis or autophagy as a major mechanism of viral release (Everett and McFadden 1999; O'Brien 1998; Roulston et al. 1999). In leafhoppers, the packaging of RGDV virions within apoptotic bodies or autophagosomes suggests that such viral particles are protected from immune pathways. Moreover, these apoptotic bodies or autophagosomes serve as vehicles for the release of progeny virions among cells.

However, these virus-induced proviral immune responses are limited and controlled. Excessive apoptosis or autophagy will certainly cause severe pathogenicity in the insect vector, even causing the death of insects, which blocks viral transmission by insects. Therefore, the balance between the viral attack and the limited immune response of insects, including apoptosis and autophagy, is achieved through convergent evolution of both virus and insect vector. In this case, the aim of the virus is to evolve efficient propagation to a high titer and nonlethal infection, which causes limited damage, thus allowing the virus to be persistently transmitted by insect vectors (Chen and Wei 2016).

Strikingly, this dynamic balance is also found between viral attack and counterattack in plants that have been infected by viruses. For example, autophagy in the plant host plays a role against viral infection in plants that are infected by the barley stripe mosaic virus (BSMV) (Table 1). However, the $\gamma$ b protein of BSMV inhibits autophagy by disrupting the interaction between ATG7 and ATG8 in a competitive manner to promote viral infection (Yang et al. 2018). In another example, autophagy suppresses TuMV infection through the autophagy cargo receptor NBR1, which targets the viral RNA silencing suppressor helper-component proteinase (HCpro) (Hafrén et al. 2018). Interestingly, TuMV also utilizes the VPg, a viral silencing suppressor, and $6 \mathrm{~K} 2$ proteins, which induce distinct types of ER-derived vesicles that are required for viral replication, to block the degradation of NBR1 and HCpro, thus limiting the antiviral capacity of autophagy (Hafren et al. 2018; Wei and Wang 2008; Wei et al. 2010). Autophagy can also restrict the establishment of CaMV infection, since NBR1 targets nonassembled and virus particle-forming capsid proteins for autophagy-dependent degradation (Hafrén et al. 2017). However, the CaMV-induced viroplasm protects against autophagic destruction by sequestering capsid proteins and by coordinating both particle assembly and storage, as described above (Hafrén et al. 2017).

In this arms race between plant viruses and their insect vectors, diverse strategies are applied by both sides to protect the survival of the insect vectors and to aid viral transmission, respectively. It is believed that the balance between viral infection and immune machinery of the insect is indicative of the relationship between other viruses and their plant hosts. This suggests that, for the balance, more immune response and cellular biology changes likely occur in insect vectors that have been infected by persistent viruses.

\section{CONCLUSIONS}

In summary, studies of various viruses and vector species have demonstrated the existence of a continuing arms race between plant virus and insect vector. During past decades, cultured cells have accelerated the investigation of the effects of plant viruses on the cell biology of the insect vectors. Therefore, numerous advances have been achieved, suggesting convergent evolution of virus and insect vector toward viral persistence. However, the cell biology of insect vectors that have been infected by plant viruses remains less-understood compared with the effects of plant viruses on plant hosts. The immune response of insect vectors to plant viruses also requires further study. The techniques of reverse genetics in insects are important for future exploration to discover cellular mechanisms that underlie the interaction between insect vector cells and plant viruses.

\section{LITERATURE CITED}

Beug, S. T., Beauregard, C. E., Healy, C., Sanda, T., St-Jean, M., Chabot, J., Walker, D. E., Mohan, A., Earl, N., Lun, X., Senger, D. L., Robbins, S. M., Staeheli, P., Forsyth, P. A., Alain, T., LaCasse, E. C., and Korneluk, R. G. 2017. Smac mimetics synergize with immune checkpoint inhibitors to promote tumour immunity against glioblastoma. Nat. Commun. 8:14278.

Blanc, S., Drucker, M., and Uzest, M. 2014. Localizing viruses in their insect vectors. Annu. Rev. Phytopathol. 52:403-425.

Bouley, S. J., Maginnis, M. S., Derdowski, A., Gee, G. V., O’Hara, B. A., Nelson, C. D., Bara, A. M., Atwood, W. J., and Dugan, A. S. 2014. Host cell autophagy promotes BK virus infection. Virology 456-457:87-95.

Carneiro, L. A., and Travassos, L. H. 2016. Autophagy and viral diseases transmitted by Aedes aegypti and Aedes albopictus. Microbes Infect. 18: 169-171.

Carrington, J. C., and Dougherty, W. G. 1987. Small nuclear inclusion protein encoded by a plant potyvirus genome is a protease. J. Virol. 61 : 2540-2548.

Chen, H., Zheng, L., Mao, Q., Liu, Q., Jia, D., and Wei, T. 2014. Development of continuous cell culture of brown planthopper to trace the early infection process of oryzaviruses in insect vector cells. J. Virol. $88: 4265-4274$.

Chen, Q., Chen, H., Jia, D., Mao, Q., Xei, L., and Wei, T. 2015b. Nonstructural protein Pns12 of rice dwarf virus is a principal regulator for viral replication and infection in its insect vector. Virus Res. 210: 54-61.

Chen, Q., Wang, H., Ren, T., Xie, L., and Wei, T. 2015a. Interaction between non-structural protein Pns10 of rice dwarf virus and cytoplasmic actin of leafhoppers is correlated with insect vector specificity. J. Gen. Virol. 96:933-938.

Chen, Q., and Wei, T. 2016. Viral receptors of the gut: Insect-borne propagative plant viruses of agricultural importance. Curr. Opin. Insect Sci. 16:9-13. 
Chen, Q., Zhang, L., Zhang, Y., Mao, Q., and Wei, T. 2017a. Tubules of plant reoviruses exploit tropomodulin to regulate actin-based tubule motility in insect vector. Sci. Rep. 7:38563.

Chen, Q., Zheng, L., Mao, Q., Liu, J., Wang, H., Jia, D., Chen, H., Wu, W., and Wei, T. 2019b. Fibrillar structures induced by a plant reovirus target mitochondria to activate typical apoptotic response and promote viral infection in insect vectors. PLoS Pathog. 15:e1007510.

Chen, Q., Zheng, L., Zhong, P., Jia, D., Liu, Y., Mao, Q., and Wei, T. 2019a. Gelsolin of insect vectors negatively regulates actin-based tubule motility of plant reoviruses. Phytopathol. Res. 1:19.

Chen, Y., Chen, Q., Li, M., Mao, Q., Chen, H., Wu, W., Jia, D., and Wei, T. 2017b. Autophagy pathway induced by a plant virus facilitates viral spread and transmission by its insect vector. PLoS Pathog. 13: e1006727.

Chen, Y., Lu, C., Li, M., Wu, W., Zhou, G., and Wei, T. 2016. Adverse effects of rice gall dwarf virus upon its insect vector Recilia dorsalis (Hemiptera: Cicadellidae). Plant Dis. 100:784-790.

Cheng, X., and Wang, A. 2016. The potyvirus silencing suppressor protein VPg mediates degradation of SGS3 via ubiquitination and autophagy pathways. J. Virol. 91:91.

Chiramel, A. I., Brady, N. R., and Bartenschlager, R. 2013. Divergent roles of autophagy in virus infection. Cells 2:83-104.

Clem, R. J. 2007. Baculoviruses and apoptosis: A diversity of genes and responses. Curr. Drug Targets 8:1069-1074.

Clem, R. J., Fechheimer, M., and Miller, L. K. 1991. Prevention of apoptosis by a baculovirus gene during infection of insect cells. Science 254:1388-1390.

del Pozo, O., and Lam, E. 2003. Expression of the baculovirus p35 protein in tobacco affects cell death progression and compromises $N$ genemediated disease resistance response to Tobacco mosaic virus. Mol. Plant-Microbe Interact. 16:485-494.

Deng, P., Wu, Z., and Wang, A. 2015. The multifunctional protein CI of potyviruses plays interlinked and distinct roles in viral genome replication and intercellular movement. Virol. J. 12:141.

Denton, D., and Kumar, S. 2015. Studying apoptosis in Drosophila. Cold Spring Harb. Protoc. 2015:609-613.

Derrien, B., Baumberger, N., Schepetilnikov, M., Viotti, C., De Cillia, J., Ziegler-Graff, V., Isono, E., Schumacher, K., and Genschik, P. 2012. Degradation of the antiviral component ARGONAUTE1 by the autophagy pathway. Proc. Natl. Acad. Sci. U.S.A. 109:15942-15946.

Drucker, M., Froissart, R., Hébrard, E., Uzest, M., Ravallec, M., Espérandieu, P., Mani, J. C., Pugnière, M., Roquet, F., Fereres, A., and Blanc, S. 2002. Intracellular distribution of viral gene products regulates a complex mechanism of cauliflower mosaic virus acquisition by its aphid vector. Proc. Natl. Acad. Sci. U.S.A. 99:2422-2427.

Elmore, S. 2007. Apoptosis: A review of programmed cell death. Toxicol. Pathol. 35:495-516.

Everett, H., and McFadden, G. 1999. Apoptosis: An innate immune response to virus infection. Trends Microbiol. 7:160-165.

Feng, Z., Xue, F., Xu, M., Chen, X., Zhao, W., Garcia-Murria, M. J., Mingarro, I., Liu, Y., Huang, Y., Jiang, L., Zhu, M., and Tao, X. 2016. The ER-membrane transport system is critical for intercellular trafficking of the NSm movement protein and tomato spotted wilt tospovirus. PLoS Pathog. 12:e1005443.

Gao, Q., Xu, W. Y., Yan, T., Fang, X. D., Cao, Q., Zhang, Z. J., Ding, Z. H., Wang, Y., and Wang, X. B. 2019. Rescue of a plant cytorhabdovirus as versatile expression platforms for planthopper and cereal genomic studies. New Phytol. 223:2120-2133.

Gray, S., Cilia, M., and Ghanim, M. 2014. Circulative, "nonpropagative" virus transmission: An orchestra of virus-, insect-, and plant-derived instruments. Adv. Virus Res. 89:141-199.

Gray, S., and Gildow, F. E. 2003. Luteovirus-aphid interactions. Annu. Rev. Phytopathol. 41:539-566.

Griffin, D. E., and Hardwick, J. M. 1999. Perspective: Virus infections and the death of neurons. Trends Microbiol. 7:155-160.

Hafrén, A., Macia, J. L., Love, A. J., Milner, J. J., Drucker, M., and Hofius, D. 2017. Selective autophagy limits cauliflower mosaic virus infection by NBR1-mediated targeting of viral capsid protein and particles. Proc. Natl. Acad. Sci. U.S.A. 114:E2026-E2035.

Hafrén, A., Üstün, S., Hochmuth, A., Svenning, S., Johansen, T., and Hofius, D. 2018. Turnip mosaic virus counteracts selective autophagy of the viral silencing suppressor HCpro. Plant Physiol. 176:649-662.

Haxim, Y., Ismayil, A., Jia, Q., Wang, Y., Zheng, X., Chen, T., Qian, L., Liu, N., Wang, Y., Han, S., Cheng, J., Qi, Y., Hong, Y., and Liu, Y. 2017. Autophagy functions as an antiviral mechanism against geminiviruses in plants. eLife 6:e23897.

Heaton, N. S., and Randall, G. 2010. Dengue virus-induced autophagy regulates lipid metabolism. Cell Host Microbe 8:422-432.
Hogenhout, S. A., Ammar, D., Whitfield, A. E., and Redinbaugh, M. G. 2008. Insect vector interactions with persistently transmitted viruses. Annu. Rev. Phytopathol. 46:327-359.

Huang, H. J., Bao, Y. Y., Lao, S. H., Huang, X. H., Ye, Y. Z., Wu, J. X., Xu, H. J., Zhou, X. P., and Zhang, C. X. 2015. Rice ragged stunt virus-induced apoptosis affects virus transmission from its insect vector, the brown planthopper to the rice plant. Sci. Rep. 5:11413.

Jackson, A. O., Dietzgen, R. G., Goodin, M. M., and Li, Z. 2018. Development of model systems for plant rhabdovirus research. Adv. Virus Res. 102:23-57.

Jan, N., and Khurshid, I. 2013. Programmed cell death or apoptosis: Do animals and plants share anything in common. Biotechnol. Mol. Biol. Rev. 3:111-126.

Jia, D., Chen, H., Zheng, A., Chen, Q., Liu, Q., Xie, L., Wu, Z., and Wei, T. 2012. Development of an insect vector cell culture and RNA interference system to investigate the functional role of fijivirus replication protein. J. Virol. 86:5800-5807.

Jia, D., Chen, Q., Mao, Q., Zhang, X., Wu, W., Chen, H., Yu, X., Wang, Z., and Wei, T. 2018. Vector mediated transmission of persistently transmitted plant viruses. Curr. Opin. Virol. 28:127-132.

Kabbage, M., Kessens, R., Bartholomay, L. C., and Williams, B. 2017. The life and death of a plant cell. Annu. Rev. Plant Biol. 68:375-404.

Komatsu, K., Hashimoto, M., Ozeki, J., Yamaji, Y., Maejima, K., Senshu, H., Himeno, M., Okano, Y., Kagiwada, S., and Namba, S. 2010. Viral-induced systemic necrosis in plants involves both programmed cell death and the inhibition of viral multiplication, which are regulated by independent pathways. Mol. Plant-Microbe Interact. 23:283-293.

Kudchodkar, S. B., and Levine, B. 2009. Viruses and autophagy. Rev. Med. Virol. 19:359-378.

Lan, H., Chen, H., Liu, Y., Jiang, C., Mao, Q., Jia, D., Chen, Q., and Wei, T. 2016a. Small interfering RNA pathway modulates initial viral infection in midgut epithelium of insect after ingestion of virus. J. Virol. 90: 917-929.

Lan, H., Wang, H., Chen, Q., Chen, H., Jia, D., Mao, Q., and Wei, T. 2016 b. Small interfering RNA pathway modulates persistent infection of a plant virus in its insect vector. Sci. Rep. 6:20699.

Lei, W., Liu, D., Li, P., and Hou, M. 2014. Interactive effects of southern rice black-streaked dwarf virus infection of host plant and vector on performance of the vector, Sogatella furcifera (Homoptera: Delphacidae). J. Econ. Entomol. 107:1721-1727.

Li, F., Zhang, C., Li, Y., Wu, G., Hou, X., Zhou, X., and Wang, A. 2018. Beclin1 restricts RNA virus infection in plants through suppression and degradation of the viral polymerase. Nat. Commun. 9:1268.

Li, S., Wang, S., Wang, X., Li, X., Zi, J., Ge, S., Cheng, Z., Zhou, T., Ji, Y., Deng, J., Wong, S. M., and Zhou, Y. 2015. Rice stripe virus affects the viability of its vector offspring by changing developmental gene expression in embryos. Sci. Rep. 5:7883.

Liu, W., Gray, S., Huo, Y., Li, L., Wei, T., and Wang, X. 2015. Proteomic analysis of interaction between a plant virus and its vector insect reveals new functions of hemipteran cuticular protein. Mol. Cell. Proteomics 14: 2229-2242.

Liu, Y., Mao, Q., Lan, H., Wang, H., Wei, T., and Chen, Q. 2018. Investigation of alimentary canal ultrastructure following knockdown of the Dicer-2 gene in planthoppers reveals the potential pathogenicity of southern rice black streaked dwarf virus to its insect vector. Virus Res. 244:117-127.

Ma, Y., Wu, W., Chen, H., Liu, Q., Jia, D., Mao, Q., Chen, Q., Wu, Z., and Wei, T. 2013. An insect cell line derived from the small brown planthopper supports replication of rice stripe virus, a tenuivirus. J. Gen. Virol. 94:1421-1425.

Mao, Q., Zheng, S., Han, Q., Chen, H., Ma, Y., Jia, D., Chen, Q., and Wei, T. 2013. New model for the genesis and maturation of viroplasms induced by fijiviruses in insect vector cells. J. Virol. 87:6819-6828.

Martinière, A., Bak, A., Macia, J. L., Lautredou, N., Gargani, D., Doumayrou, J., Garzo, E., Moreno, A., Fereres, A., Blanc, S., and Drucker, M. 2013. A virus responds instantly to the presence of the vector on the host and forms transmission morphs. eLife 2:e00183.

Mitchell, J. K., and Friesen, P. D. 2012. Baculoviruses modulate a proapoptotic DNA damage response to promote virus multiplication. J. Virol. 86:13542-13553.

Movahed, N., Patarroyo, C., Sun, J., Vali, H., Laliberté, J. F., and Zheng, H. 2017. Cylindrical inclusion protein of turnip mosaic virus serves as a docking point for the intercellular movement of viral replication vesicles. Plant Physiol. 175:1732-1744.

Nakamoto, M., Moy, R. H., Xu, J., Bambina, S., Yasunaga, A., Shelly, S. S., Gold, B., and Cherry, S. 2012. Virus recognition by Toll-7 activates antiviral autophagy in Drosophila. Immunity 36:658-667. 
Nakasuji, F., and Kiritani, K. 1970. Ill-effects of rice dwarf virus upon its vector, Nephotettix cincticeps Uhler (Hemiptera: Deltocephalidae), and its significance for changes in relative abundance of infected individuals among vector populations. Appl. Entomol. Zool. 5:1-12.

Nasu, S. 1963. Studies on some leafhoppers and planthoppers which transmit virus diseases of rice plant in Japan. Bull. Kyushu Agric. Exp. Stn 8:153-349.

Nyalugwe, E. P., Barbetti, M. J., Clode, P. L., and Jones, R. A. C. 2016. Programmed cell death pathways induced by early plant-virus infection are determined by isolate virulence and stage of infection. Plant Pathol. 65:1518-1528.

O’Brien, V. 1998. Viruses and apoptosis. J. Gen. Virol. 79:1833-1845.

O’Neill, K., Olson, B. J., Huang, N., Unis, D., and Clem, R. J. 2015. Rapid selection against arbovirus-induced apoptosis during infection of a mosquito vector. Proc. Natl. Acad. Sci. U.S.A. 112:E1152-E1161.

Ogada, P. A., Maiss, E., and Poehling, H. M. 2012. Influence of tomato spotted wilt virus on performance and behaviour of western flower thrips (Frankliniella occidentalis). J. Appl. Entomol. 27:1-10.

Pei, J., Zhao, M., Ye, Z., Gou, H., Wang, J., Yi, L., Dong, X., Liu, W., Luo, Y., Liao, M., and Chen, J. 2014. Autophagy enhances the replication of classical swine fever virus in vitro. Autophagy 10:93-110.

Roulston, A., Marcellus, R. C., and Branton, P. E. 1999. Viruses and apoptosis. Annu. Rev. Microbiol. 53:577-628.

Rubinstein, G., and Czosnek, H. 1997. Long-term association of tomato yellow leaf curl virus with its whitefly vector Bemisia tabaci: Effect on the insect transmission capacity, longevity and fecundity. J. Gen. Virol. 78:2683-2689.

Shelly, S., Lukinova, N., Bambina, S., Berman, A., and Cherry, S. 2009. Autophagy is an essential component of Drosophila immunity against vesicular stomatitis virus. Immunity 30:588-598.

Sidhu, J. S., Mann, R. S., and Butter, N. S. 2009. Deleterious effects of Cotton leaf curl virus on longevity and fecundity of whitefly, Bemisia tabaci (Gennadius). J. Entomol. 6:62-66.

Sundström, J. F., Vaculova, A., Smertenko, A. P., Savenkov, E. I., Golovko, A., Minina, E., Tiwari, B. S., Rodriguez-Nieto, S., Zamyatnin, A. A., Jr., Välineva, T., Saarikettu, J., Frilander, M. J., Suarez, M. F., Zavialov, A., Ståhl, U., Hussey, P. J., Silvennoinen, O., Sundberg, E., Zhivotovsky, B., and Bozhkov, P. V. 2009. Tudor staphylococcal nuclease is an evolutionarily conserved component of the programmed cell death degradome. Nat. Cell Biol. 11:1347-1354.

Tu, Z., Ling, B., Xu, D., Zhang, M., and Zhou, G. 2013. Effects of southern rice black-streaked dwarf virus on the development and fecundity of its vector, Sogatella furcifera. Virol. J. 10:145.

Uren, A. G., O'Rourke, K., Aravind, L. A., Pisabarro, M. T., Seshagiri, S., Koonin, E. V., and Dixit, V. M. 2000. Identification of paracaspases and metacaspases: Two ancient families of caspase-like proteins, one of which plays a key role in MALT lymphoma. Mol. Cell 6:961-967.

Vaidyanathan, R., and Scott, T. W. 2006. Apoptosis in mosquito midgut epithelia associated with West Nile virus infection. Apoptosis 11: 1643-1651.

van Doorn, W. G., Beers, E. P., Dangl, J. L., Franklin-Tong, V. E., Gallois, P., Hara-Nishimura, I., Jones, A. M., Kawai-Yamada, M., Lam, E., Mundy, J., Mur, L. A., Petersen, M., Smertenko, A., Taliansky, M., Van
Breusegem, F., Wolpert, T., Woltering, E., Zhivotovsky, B., and Bozhkov, P. V. 2011. Morphological classification of plant cell deaths. Cell Death Differ. 18:1241-1246.

Wan, G., Jiang, S., Wang, W., Li, G., Tao, X., Pan, W., Sword, G. A., and Chen, F. 2015. Rice stripe virus counters reduced fecundity in its insect vector by modifying insect physiology, primary endosymbionts and feeding behavior. Sci. Rep. 5:12527.

Wang, H., Gort, T., Boyle, D. L., and Clem, R. J. 2012. Effects of manipulating apoptosis on Sindbis virus infection of Aedes aegypti mosquitoes. J. Virol. 86:6546-6554.

Wang, L. L., Wang, X. R., Wei, X. M., Huang, H., Wu, J. X., Chen, X. X., Liu, S. S., and Wang, X. W. 2016. The autophagy pathway participates in resistance to tomato yellow leaf curl virus infection in whiteflies. Autophagy 12:1560-1574.

Wei, T., Huang, T. S., McNeil, J., Laliberté, J. F., Hong, J., Nelson, R. S., and Wang, A. 2010. Sequential recruitment of the endoplasmic reticulum and chloroplasts for plant potyvirus replication. J. Virol. 84:799-809.

Wei, T., and Li, Y. 2016. Rice reoviruses in insect vectors. Annu. Rev. Phytopathol. 54:99-120.

Wei, T., Miyazaki, N., Uehara-Ichiki, T., Hibino, H., Shimizu, T., Netsu, O., Kikuchi, A., Sasaya, T., Iwasaki, K., and Omura, T. 2011. Threedimensional analysis of the association of viral particles with mitochondria during the replication of Rice gall dwarf virus. J. Mol. Biol. 410:436-446.

Wei, T., Shimizu, T., Hagiwara, K., Kikuchi, A., Moriyasu, Y., Suzuki, N., Chen, H., and Omura, T. 2006. Pns 12 protein of Rice dwarf virus is essential for formation of viroplasms and nucleation of viral-assembly complexes. J. Gen. Virol. 87:429-438.

Wei, T., and Wang, A. 2008. Biogenesis of cytoplasmic membranous vesicles for plant potyvirus replication occurs at endoplasmic reticulum exit sites in a COPI- and COPII-dependent manner. J. Virol. 82: 12252-12264.

Wu, W., Zheng, L., Chen, H., Jia, D., Li, F., and Wei, T. 2014. Nonstructural protein NS4 of Rice stripe virus plays a critical role in viral spread in the body of vector insects. PLoS One 9:e88636.

Yang, M., Zhang, Y., Xie, X., Yue, N., Li, J., Wang, X. B., Han, C., Yu, J., Liu, Y., and Li, D. 2018. Barley stripe mosaic virus $\gamma \mathrm{b}$ protein subverts autophagy to promote viral infection by disrupting the ATG7-ATG8 interaction. Plant Cell 30:1582-1595.

Yang, X., Zhang, T., Chen, B., and Zhou, G. 2017. Transmission biology of rice stripe mosaic virus by an efficient insect vector Recilia dorsalis (Hemiptera: Cicadellidae). Front. Microbiol. 8: 2457.

Zhao, P., Sun, X., Li, P., Sun, J., Yue, Y., Wei, J., Wei, T., and Jia, D. 2019. Infection characteristics of rice stripe mosaic virus in the body of the vector leafhoppers. Front. Microbiol. 9:3258.

Zheng, L., Chen, H., Liu, H., Xie, L., and Wei, T. 2015. Assembly of viroplasms by viral nonstructural protein Pns9 is essential for persistent infection of rice gall dwarf virus in its insect vector. Virus Res. 196: 162-169.

Zhou, X., Lin, W., Sun, K., Wang, S., Zhou, X., Jackson, A. O., and Li, Z. 2019. Specificity of plant rhabdovirus cell-to-cell movement. J. Virol. 93:e00296-19. 